\title{
A vacina contra HPV e o câncer de colo de útero: desafios para a sua incorporação em sistemas de saúde
}

\author{
Hillegonda Maria Dutilh Novaes
}

O câncer de colo de útero ainda é um importante problema de saúde populacional, particularmente nas regiões mais pobres do mundo, apesar de programas para detecção precoce com base no exame de Papanicolaou terem sido propostos há mais de 50 anos $^{1,2}$. Esses programas mostraram-se custo-efetivos, desde que alcancem elevada cobertura populacional na população feminina e façam parte de programas de atenção à saúde da mulher, com adequada indicação e realização do exame, coleta e análise do material, entrega do resultado e encaminhamento terapêutico oportuno e correto, quando necessário. O que não acontece em muitos contextos. Envolvem um número diversificado de níveis de atenção e muitos profissionais da atenção primária, serviços de laboratório e serviços especializados (ginecologia, oncologia). Sendo assim, a frustração quanto aos resultados insatisfatórios de controle de um câncer que do ponto de vista científico e técnico poderia ser prevenido é compartilhado por muitos, o que explica a intensa repercussão observada, no meio científico e fora dele, da recente aprovação para comercialização de vacinas contra a infecção por HPV. O texto de Juan Gervás, médico espanhol que trabalha em atenção primária, representa a opinião de um desses grupos.

As vacinas se constituem em tecnologias consideradas prioritárias para a saúde das populações, e dois fatores podem ser destacados como importantes para as transformações recentes nesse setor: desenvolvimento científico e tecnológico e de inovação (genômica, proteômica) e a proposição pelas organizações internacionais de fundos globais de financiamento das vacinas, com o desenvolvimento de novas vacinas, investimentos crescentes por parte das indústrias de medicamentos e incorporação de novas vacinas nos sistemas de saúde ${ }^{3}$. Observase nas vacinas as mesmas dinâmicas que caracterizam atualmente os processos de produção de tecnologias e sua incorporação e difusão nos sistemas de saúde, porém com uma tendência para o encurtamento dos tempos entre a demonstração da segurança e eficácia, realização de estudos de custoefetividade, aprovação para o mercado, difusão em escala populacional e incorporação em programas de saúde ${ }^{4}$.

A vacina contra o HPV se constitui em um caso particularmente interessante. Uma vacina profilática contra infecção persistente de sorotipos de HPV, reconhecidamente associados ao desenvolvimento do câncer de colo uterino (causa necessária mas não suficiente). Estão aprovadas e em fase final de aprovação pela vigilância sanitária de vários países duas vacinas com características semelhantes. As vacinas foram submetidas a ensaios clínicos multi-cêntricos internacionais de fase III (foram incluídas nos ensaios mulheres de todos os continentes, menos a África, com importante participação brasileira), com resultados bastante favoráveis até agora quanto à eficácia (para lesões cervicais associadas à infecção persistente) e segurança, e vários estudos em populações específicas estão em andamento ${ }^{5,6}$. So far so good.

Como os resultados sobre a morbidade e mortalidade do câncer de colo de útero somente ocorrerão após um longo período de latência, foram e estão sendo desen- 
volvidos diversos estudos de modelização da epidemiologia da doença e análises de custo-efetividade. Nessas análises são comparadas a situação atual, para determinados contextos epidemiológicos e de atenção à saúde, e diferentes alternativas de rastreamento do câncer de colo de útero e implementação de programa de imunização universal de meninas pré-adolescentes. De maneira geral, os resultados desses estudos se mostram otimistas, apresentando resultados que indicam estimativas para razões incrementais possivelmente custoefetivas ${ }^{7,8,9,10}$.

No entanto, na maioria dos estudos, para se chegar a valores que podem ser considerados custo-efetivos, o preço da vacina teria que ser muito menor do que é atualmente, o que parece distante de ser alcançado no momento. O mais interessante, contudo, quando então se mostram muito pertinentes as preocupações de Juan Gervás, é que a maioria das análises não leva em conta as dificuldades já conhecidas no desempenho dos programas de rastreamento do câncer (mencionadas quando da valorização da vacina), e praticamente ignoram as dificuldades imensas, tanto operacionais quanto culturais, a serem enfrentadas na implantação adicional de um programa de imunização universal em meninas pré-adolescentes nos sistemas de saúde.

Nesse sentido, ainda que os estudos de custo-efetividade venham se mostrando uma forma de produção de conhecimento que pode ser útil no apoio às decisões dos gestores, deve-se reconhecer que as decisões em si, e sua tradução em iniciativas políticas e técnicas, passam por processos muito mais complexos e abrangentes ${ }^{11,12}$.

\section{Referências}

1. Althuis MD, Dozier JM, Anderson WE et al. Global trends in breast cancer incidence and mortality 1993-1997. Int J Epidemiol 2005; 34(2): 405-12.

2. Fonseca LS, Ramaciotti AS, Eluf Neto J. Mortality trends from uterine cervical cancer in the city of São Paulo from 1980 to 1999. Cad Saude Publica 2004; 20(1): 136 42.

3. Hardon A, Blume S. Shifts in global immunization goals (1984-2004): unfinished agendas and mixed results. Soc Sci Med 2005; 60: 345-56.

4. Novaes HMD, Carvalheiro JR. Ciência, tecnologia e inovação em saúde e desenvolvimento social e qualidade de vida: teses para debate. Cienc Saúde Coletiva 2007; 12: 1841-49.

5. Cutts FT, Francsechi S, Goldie S et al. Human papillomavirus and HPV vaccines: a review. Bull WHO 2007; 85(7): 719-26.

6. Zimet GD, Shew ML, Kahn JA. Appropriate use if cervical cancer vaccine. Annu Rev Med 2008; 59: 223-36.
7. Goldie S, Goldhaber-Fiebert JD, Garnett GP. Public health policy for cervical cancer prevention: the role of decision science, economic evaluation and mathematical modeling. Vaccine 2006; S3: 155-63.

8. Goldie S, Kim JJ, Kobus K et al. Costeffectiveness of HPV 16, 18 vaccination in Brazil. Vaccine 2007; 25: 6257-70.

9. Diaz M, Kim JJ, Albero G et al. Health and economic impact of HPV 16 and 18 vaccination and cervical cancer screening in India. Br J Cancer 2008; 99(2): 230-8.

10. Gutiérrez-Delgado C, Báez-Mendoza C, González-Pier E. Relación costo-efetividad de las intervenciones preventivas contra él cáncer cervical en mujeres mexicanas. Salud Publica de México 2008; 50: 107-17.

11. Pollack AE, Balkin M, Edouard L, et al. Ensuring access tto HPV vaccines through integrated services: a reproductive health perspective. Bull WHO 2007; 85: 57-63.

12. Roughhead EE, Gilbertt AL, Vitry AI. The Australian funding debate on quadrivalent HPV vaccine: a case study for the national pharmaceutical policy. Health Policy 2008 May 9. [Epub ahead of print]. 